Kathrin Rosenfield

\title{
Quecksilbersachen: J.G. Rosa und Curt Meyer-Clason
}

\author{
Im Dialog mit B. Zilly, P. Perazzo und Taís Lucas
}

\section{Einleitung}

Die Interaktion zwischen J.G. Rosa, dem Autor metaphysischer Visionen, und Meyer-Clason, dem realistischen und pragmatischen Übersetzer, ist ein Schachspiel aus Paradoxien, die nicht einmal die Reihe der Komplimente durch den Autor abmildern kann. Sie fällt gänzlich in den Bereich der Quecksilbersachen, der brasilianischen Ambiguität und Höflichkeit - aus Gesten und Attitüden, die das Werk und das Leben im Dämmerlicht der komplexen Beziehungen halten, die sich zwischen Fakt und Fiktion, Poesie und Wahrheit weben.

Bereits die Wege der zwei Männer im geopolitischen Sertão der Epoche tragen einen Hauch Ironie in sich. Während Meyer-Clason 1936 nach Brasilien übersiedelt, beginnt die zukünftige Frau von Rosa, Aracy Moebius, im Hamburger Konsulat zu fungieren, wo sie später den Verlobten dazu inspirieren wird, seine diplomatische Karriere aufs Spiel zu setzen, um gefährdete Juden vor dem dritten Reich zu retten. Diese Erfahrungen mit dem Horror des Sertão, der die Welt ist, schwingen im großen rosianischen Roman mit, den Meyer-Clason übersetzen wird.

Mit Grande sertão: veredas kreierte Rosa einen ungewöhnlichen Rahmen im Bereich der literarischen Schönheit. Seine Figur Riobaldo ist auch eine wichtige imaginäre Erinnerungsfigur, die eine schwierige moralische Aufgabe beschäftigt: die Barrieren der Selbsttäuschung aufzuheben und die Untersuchung der Fakten durch Selbsterfahrung und die durch die Fakten mögliche persönliche Entwicklung zu reinigen. Das Narrativ von Riobaldo ist ein seltenes Beispiel der ethischen Rechtschaffenheit, die sich nicht darauf reduziert, von objektiven Traumata zu sprechen, sondern einen Teil der Schuld an den Leiden anzuerkennen versucht, die immer die Intentionen des Individuums übersteigen, gleichzeitig aber dennoch in unzähligen (unbedachten, unbewussten, nicht direkt beabsichtigten) Gesten der Individuen auftauchen, wodurch das Kollektiv geformt wird, das erstaunliche Taten hervorbringt.

Aus diesem Grunde ist es möglich, so zu tun, als hätte man keine Kenntnisse über eine Reihe von Erzählungen über den umgekehrten Weg von Meyer-Clason in Brasilien - die Gerüchte um Spionage im Dienste des dritten Reiches, die seit Jahrzehnten zirkulieren, ohne dass irgendwer es wagt zu sagen, ob es sich um

כ Open Access. @ 2020 Kathrin Rosenfield, publiziert von De Gruyter. @) BY-Nc-ND Dieses Werk ist lizenziert unter der Creative Commons Attribution-NonCommercial-NoDerivatives 4.0 Lizenz. https://doi.org/10.1515/9783110677713-012 
Fakten oder Fiktion handelt. Sie rücken das Leben von Rosas Übersetzer von 1930 bis 1945 in ein beunruhigendes Licht - ein Licht, das wahr oder falsch sein kann. Hier kann zumindest dasjenige hervorgehoben werden, was man weiß und was ermittelt wurde. Auf diese Weise können die beschämenden Dinge, die sich vielleicht oder vielleicht auch nicht auf die Erinnerung an Meyer-Clason auswirken, von der Schwelle des Nicht-Gesagten oder Dazwischen-Gesagten angestoßen werden. Zu sehen, was wahr ist oder nicht, hat Relevanz für uns Kritiker und Intellektuelle die wir Sinn und Wert der Literatur und der Übersetzung, des direkten und indirekten Engagements der Poesie ${ }^{1}$ diskutieren wollen.

Wir alle wissen, dass es seit 1998 eine historische Forschung gibt, die unter der Leitung von Prof. Tucci Carneiro von Priscila Perazzo entwickelt wurde. In ihrer Forschung für die Erarbeitung ihrer Masterthese fand Perazzo in jener Zeit ein fotographisches Dossier, das zu diesem Zeitpunkt unveröffentlicht war und das Meyer-Clason als typischen Spion der Nazis in Brasilien darstellte. Priscila hatte nicht das geringste Interesse daran, Meyer-Clason bloßzustellen (sie war sich der kulturellen Relevanz des Autors kaum bewusst), sie fand lediglich die Dokumente, die 1942 aufgrund der Mitwirkung in einem nationalsozialistischen Spionagenetz zur Verhaftung von Meyer-Clason führten.

Weniger bekannt ist die jüngere These von Taís Campelo $\mathrm{Lucas}^{2}$, die diesen Verdacht verstärkt und beabsichtigt, diesen Fall weiter zu untersuchen.

Beide Untersuchungen enthalten momentan eine Reihe von Lücken, die es verhindern, Schlüsse zu ziehen und bedürfen der Klärung. Meine Arbeit beschränkt sich darauf, das Gewirr der widersprüchlichen Informationen zu erfassen. In erster Linie interessiert mich lediglich die Art und Weise, wie wir unsere Wahrnehmung von ihm, Meyer-Clason., lenken: welche Elemente - verfügbar oder nicht - wir sehen, welche wir übersehen. Mit welcher Geschwindigkeit oder Ziellosigkeit verarbeiten wir die existierenden Erzählungen, wie sorgfältig oder oberflächlich ist unsere Art, die Teile des (unvollständigen) Puzzles, das wir besitzen, zusammenzufügen. Ein jüngstes Detail bestärkt die Bedeutung der Suche nach Wahrheit: Priscila Perazzo wurde während der Verteidigung ihrer Masterarbeit über die wichtige Rolle, die Meyer-Clason in den deutsch-brasilianischen Beziehungen erfüllte, informiert. Es war der damalige Direktor des Goethe-Insti-

1 Ich erwähne das Engagement, weil es eine Form ist, die Ethik der Poesie zu thematisieren. Darüber hinaus gibt Rosa interessante Erklärungen über sein Verständnis poetischen Engagements, die denen des Konzepts von R. Musil sehr ähnlich sind, an dem ich momentan arbeite.

2 Taís Lucas: Nazismo d'além mar: conflitos e esquecimento. Tese de doutorado, UFRGS Porto Alegre 2011, S. 182-183. Os dados são do Relatório do DOPS. Porto Alegre, am 20. Mai 1942. DOPS, Aktennr. 36.691: Rede de Espionagem no Rio Grande do Sul ou Nazismo no Rio Grande do Sul, AESP. 
tuts in São Paulo, der seine Verwunderung aufgrund seines Unwissens über diesen möglichen Abschnitt in Meyer-Clasons Geschichte ausdrückte und in diesem öffentlichen Prüfungskomitee von der wichtigen Rolle berichtete, die der Übersetzer zusammen mit dem Goethe-Institut in Lisboa während der Diktatur Salazars in dem Land hatte. Dies weckte das Interesse der großen paulistanischen Presse, die die Forscherin unzählige Male aufgriff und auf peinliche Art und Weise eine objektive Antwort erzwang, dass Meyer-Clason tatsächlich als nationalsozialistischer Spion in Brasilien agiert hätte. Diese Neuigkeiten gingen sogar in Deutschland in einer Ausgabe des Spiegels um und verstärkten die Verlegenheit, die von der ungewöhnlichen Nachricht provoziert wurde, dass Priscila nicht direkt mit Meyer-Clason sprechen konnte, da dieser nicht auf ihre Interviewanfragen antwortete. Aus diesen Gründen gab Priscila das Forschungsprojekt auf und nahm eine umsichtige Position der Aufhebung des Schuldspruchs und des Skeptizismus hinsichtlich der Fakten ein, die zuvor so klar erschienen waren. Sie respektierte sogar die Erklärung Meyer-Clasons im Spiegel, dass dieser von der brasilianischen Polizei gefoltert worden war, damit er seine Beteiligung an jenem Spionagenetzwerk gestand. Was Priscila nicht wusste, war das Detail, welches Juliane, die Tochter Meyer-Clasons, mir in Berlin während eines Kolloquiums erzählte: Derselbe Direktor des Goethe-Instituts (Dieter Strauß), der Priscila zu soviel Skeptizismus und Umsicht angehalten hatte, hält heute Vorträge in Buchhandlungen und kulturellen Events über die Nazivergangenheit von Meyer-Clason und verbreitet Informationen, die, Juliane zufolge, durchweg phantastisch und unwahr sind.

In diesem Moment befinde ich mich in der Position des Kritikers, der über eine Reihe widersprüchlicher Informationen verfügt und auch die Auffassung bekannt macht, die wir von der deutschen Übersetzung von Meyer-Clason haben. In ihr gibt es eine Reihe von Varianten, die Aufmerksamkeit auf sich ziehen und Interpretation verlangen, zum Beispiel die Stärkung des lokalen Realismus, der die Geschichte im minerischen Sertão verortet und die universalistischen, metaphysischen Hinweise unterdrückt, die das Problem des Schlechten und des Teufels auf die Welt als Ganzes ausdehnen. Ich beginne daher meine Bestandsaufnahme in einem Dialog mit den Übersetzern, die hier von Berthold Zilly und Fabio Chiqueto repräsentiert werden.

\section{Die antimetaphysische Übersetzung und Meyer-Clasons Erklärungen}

Es ist allgemein bekannt, dass in der deutschen Übersetzung von Grande sertão: veredas die Unterdrückung großer Teile der spirituellen und metaphyischen Be- 
deutungen vorherrschend ist, die eine so wichtige Rolle im Roman erfüllen und in der Vision, die der Autor Rosa von der Poesie, vom Leben und vom eigenen Werk hat. Indem ich mich auf Arbeiten anderer Kritiker stütze, kann ich an dieser Stelle zumindest einen einmaligen Aspekt der übersetzerischen Möglichkeiten von Meyer-Clason herausheben, die unzweideutige Intention des Autors, den regionalen Realismus hervorstechen zu lassen, zum Nachteil des spirituellen oder metaphysischen Universalismus, der Guimarães Rosa so wichtig war.

Im Laufe dieses Artikels werde ich zunächst auf die Zusammenfassung von Fabio Chiqueto zurückgreifen, die die häufigen «Verirrungen in der Übersetzung» von Meyer-Clason unterstreicht, um anschließend zu den Eindrücken Berthold Zillys zu kommen. Die übersetzerischen Verirrungen sind entweder «semantischer Natur, stilistischer oder gar ideologischer». ${ }^{3}$ In ihrer Gänze verdeutlichen sie die Tatsache, dass Meyer-Clason sich weigert, das reiche Spektrum metaphysischer Vorschläge zu beachten, den poetischen und metaphysischen Schein, der den referentiellen Sinn der regionalen Termini transzendiert. Meyer-Clason sucht nach der konkreten lokalen Färbung und beraubt die rosianischen Figuren ihrer einzigartigen allegorischen Dichte (der Sertão wird zu einer regionalen Realität, anstatt als hochgradig ambiger Topos zu fungieren, der sich in imaginären Figuren zweiten und dritten Grades entfaltet: der Sertão, der hier ist und dort, weit weg und weitab ab von uns, ist der Sertão die Welt).

Chiqueto hat gezeigt, dass diese Tendenz dem Ansatz von Meyer-Clason inhärent ist, sowie dass der Übersetzer seine Schwierigkeit, mit Rosas Sprache zu arbeiten, explizit zum Ausdruck brachte, und zitiert die Worte des Autors selbst:

In einem ersten Moment, nach der Lektüre des Grande sertão -, sagt der Übersetzer -, dachte ich bei mir: Entweder existiert diese Sprachw nicht oder ich war niemals in Brasilien. Natürlich hatte ich Trilhas do sertão von Cavalcanti Proença gelesen, was mir jedoch sehr geholfen hat, war die Einleitung der englischen Version des GSV, von Jorge Amado. Dennoch erkenne ich an, dass die Manie des Amerikaners, die Sprache dermaßen auf den commonsense zu reduzieren, auf eine absolute Rationalität, eine originalgetreue Übersetzung von Rosas Werk nicht erlaubte. Das herrliche Bild - Dia das Abelhas Brancas (dt. wörtl. Tag der weißen Bienen) - wurde übersetzt als A red letter day (Meyer-Clason 1968). ${ }^{4}$

Während er gleichzeitig Unmut über die amerikanische Übersetzung äußert, bevorzugt Meyer-Clason eben diese Richtung, ${ }^{5}$ was den Erwartungen Rosas ent-

3 Fábio Luís Chiqueto Barbosa: Leituras de Grande sertão: veredas: sua tradução alemã e a correspondência de Guimarães Rosa com seu tradutor alemão Curt Meyer-Clason. In: Signótica 22/1 (2010), S. 57-68, hier S. 61.

4 Nach Chiqueto Barbosa: Leituras de Grande sertão: veredas, S. 64.

5 Ebda., S. 65. 
gegensteht, der sich von dem deutschen Übersetzer eine besondere Sensibilität für die poetische Dichte erhoffte, die die physische mit der metaphysischen Erfahrung verbindet. Es gibt Gründe dafür, anzunehmen, dass diese Unterdrückung «der Überlagerung mehrerer Sinnebenen» nicht, wie Chiqueto sagt, ein [nur] teilweise bewusster Prozess von Meyer-Clasons Seite ist, ${ }^{6}$ sondern dass dieser notwendigerweise aus der defensiven Einstellung hervorgeht, die Meyer-Clason der metaphysischen Dimension des rosianischen Werks gegenüber einnimmt.

Wenn dieser Widerstand gegen die zentrale Charakteristik des rosianischen Werkes bereits seltsam erscheint, dann ist die Angst des Autors, das Werk Rosas mit der Vorstellungswelt des Nationalsozialismus verknüpft zu sehen, noch merkwürdiger (was sicherlich dem metaphysischen Hang des brasilianischen Autors gerecht werden würde).

Zitiert wird an dieser Stelle Meyer-Clasons Erklärung in einem Interview, dass er Lísia Dornelles gab, in dem er den regionalen Realismus der Übersetzung rechtfertigt:

\begin{abstract}
Aufgrund des Traumas, welches durch das durch das zwölf Jahre lang existierende dritte Reich verursacht worden war, blieb das Volk tief gezeichnet und hat dies bis heute nicht ganz überwunden. Von daher hat der Leser, in erster Linie der kritische Leser, große Angst vor der Metaphysik. Die Fiktion mit metaphysischem Tenor ruft heute nicht mehr die gleiche Attraktivität hervor wie vor zwanzig Jahren, in Zeiten von Hermann Hesse und Thomas Mann. Was den deutschen Leser an Rosas Werk begeistert, ist die Farbe, die Plastizität, die Kraft, die zyklische Vitalität., 8
\end{abstract}

Diese Stellungnahme ist auf mehreren Ebenen befremdlich wie aufschlussreich: erstens wegen der missbräuchlichen Generalisierungen (jeder Leser oder Kritiker hätte nach dem Nationalsozialismus Angst vor der Metaphysik), zweitens wegen der Unachtsamkeit dieser Distanzierung von der (vorgeblich nationalsozialistischen) Metaphysik und der (viel näher an der nationalsozialistischen Vorstellungswelt gelegenen) Alternative des «plastischen» Realismus, der «das Publikum für die Kraft und zyklische Vitalität begeistern» soll. Denn Kraft und Vitalität sind durchaus Begriffe, die sehr nah am neodarwinistischen Gusto und der Propaganda des dritten Reichs liegen - um ein Vielfaches näher als die rosianische Metaphysik. Ebenfalls seltsam ist auf einer dritten Ebene auch die plumpe Formulierung, mit der Meyer-Clason die Metaphysik mit dem National-

6 Ebda.

7 Curt Meyer-Clason: Saudade do Brasil faz um grande tradutor. Interview mit Lisia Dornelles. In: Correio da Manhã (1.1.1968).

8 Unsere Hervorhebungen, vgl. Chiqueto Barbosa: Leituras de Grande sertão: veredas, S. $65 f$. 
sozialismus assoziiert oder seine Präferenz für die Kraft und Vitalität der realistischen Bilder des Landes kundgibt. Dieses Spektrum unterstreicht einen hohen Grad an fehlendem Bewusstsein bezüglich des deutschen literarisch-philosophischen Denkens und bezüglich seiner Sprachregister (was einen symptomatischreaktiven Widerstand nahelegt). In diesem Interview wird die Gleichgültigkeit offensichtlich, wenn nicht gar die Verachtung der europäischen neomystischen Tradition, die Rosa so sehr anzog und die unter so vielen Künstlern und Denkern während der ersten Hälfte des 20. Jahrhunderts ${ }^{9}$ in Mode war. Die Angst, die Meyer-Clason als Allheilmittel für das nationalsozialistische Trauma vorschlägt, hat etwas von einem persönlichen Symptom und scheint keine angemessene übersetzerische Variante zu sein - weder, um das rosianische Werk in die deutsche Sprache zu übermitteln, noch, um die ideologische Entfremdung des Nationalsozialismus zu überwinden. Denn die Neo-Mystiker, die Rosa kannte, nachahmte und bewunderte, sind frei von jeglicher Verbindung zur nationalsozialistischen Ideologie: Kafka und M. Buber, abgesehen von W. Benjamin und G. Sholem und ganz zu Schweigen von dem breiten Kontingent der (künstlerischen, wissenschaftlichen, philosophischen und pädagogischen) Intellektualität, die rein garnichts mit dem Nationalsozialismus zu tun hat. Rilke und Heinrich Mann, Hofmannsthal, Musil und Altenberg, Rudolph Steiner und Klages, Maeterlinck und Ricarda Huch, Mauthner, Gustav Landauer und Einstein sie alle debattierten auf der Grundlage intensiver neomystischer Reflexionen, und obwohl Steiner und Klages des Antisemitismus verdächtigt wurden, läuft das Spektrum dieser Autoren keineswegs Gefahr, mit dem Nationalsozialismus in Verbindung gebracht zu werden. Es gab in der Tat Denker, deren Ideen durch die nationalsozialistische Propaganda verfälscht wurde (Nietzsche, Moeller van den Bruck und viele andere). Es gab auch Neo-Mystiker und Denker des mittelalterlichen Mystizismus, die anfangs mit dem vagen, von den Ideologen des Nationalsozialismus geteilten Nationalismus sympathisierten (man denke an den Kreis um Stefan George, eines mystisch-nationalistischen Umfelds, innerhalb dessen E. Kantorowicz seine berühmten Bücher The King's Two Bodies und Frederico $I I^{10}$ schrieb.

9 Wir denken lediglich an Wassily Kandinsky (O espiritual na arte), und an Frank Lloyd Wright, dessen Spiralprojekt des Guggenheim Museum die theosophischen Fantasien von Hilla Rebay anregte, die für ihren Teil vom Mystizismus des Georgji Gurdjieff und Kandinsky inspiriert wurde. Vgl. Roger Friedland und Harold Zellmann: The Fellowship. The Untold Story of Frank Lloyd Wright and the Taliesin Fellowship. New York: Harper Collins 2006, S. 378.

10 Ernest Kantorowics: The King's Two Bodies. A Study in Mediaeval Political Theology. Princeton: Princeton University Press 1957 und ders.: Kaiser Friedrich der Zweite. Berlin: Georg Bondi 1927. Man muss kein Kenner all dieser Autoren sein, die zahlreiche Felder abdecken - von der Philoso- 
Wie kann man die Bemerkung des Übersetzers interpretieren? Es ist wahrscheinlich, dass es in erster Linie Zeugnis seiner auf Handel und Unternehmensführung und weniger auf das intellektuelle Leben in den Humanwissenschaften ausgerichteten Karriere ist. Douglas' Beitrag zum Berliner Kongress zeigte, dass Meyer-Clason im Gefängnis in die Lektüre und das intellektuelle Leben eingeführt wurde und dass sein Mentor auf der Ilha Grande während seiner fünf Jahre im Gefängnis eine Vorliebe für konservative Autoren hatte. Vielleicht hat sich das Bild der deutschen Kultur in dieser späteren Koexistenz gefestigt, was zu einer Reihe von Missverständnissen und Ungenauigkeiten in der Vision des geistigen und intellektuellen Lebens Deutschlands, wie Rosa es begriff, führte. In dem Interview offenbart der Übersetzer, dass seine Übersetzung die möglichen bloßstellenden Assoziationen beseitigen wollte, die er, Meyer-Clason, in Rosas Arbeit zu projizieren scheint. Deshalb hat er möglicherweise im Interview diese etwas pedantische Position eines Pädagogen angenommen, der Rosa von Verbindungen mit der faschistischen (angeblich mystischen) Ideologie reinigte - Verbindungen, die ein Kenner Rosas und der deutschen Kultur nie knüpfen würde. Es ist im Allgemeinen nicht notwendig, 〈den Leser und den Kritiker〉 vor möglichen zweideutigen Assoziationen zwischen der obskurantistischen Mythomanie der NS-Propaganda (deren nächtliche und Begräbniskulte pseudomystische Scheinkulte boten) und der philosophischen, literarischen und künstlerischen Debatte über Metaphysik und Mystik in Deutschland und Österreich zu schützen. Kein Leser würde die oben genannten Autoren mit den trivialen Propagandaersatzmitteln der Nazi-Ästhetik in Verbindung bringen. Die vage Nostalgie nach Einheit und spiritueller Erhebung der Mitläufer hat wenig bis garnichts mit dem metaphysischen Geist von Rosa, Rilke oder Hofmannsthal und vielen anderen Neomystikern zu tun. Die Angst von Meyer-Clason kann nur in einem Kopf voller ideologischer Unsicherheiten keimen, die dazu führen, dass die religiöse, mystische oder metaphysische Dimensi-

phie über die Theologie bis hin zur Anthropologie, sowie der Literaturgeschichte. Es gibt eine Reihe klassischer und zeitgenössischer Werke, die die Beziehungen zwischen der kulturellen, politischen Kritik und dem Mystizismus in der ersten Hälfte des 20. Jahrhunderts in ihrer Tiefe untersuchen, mit spezifischem Fokus in den Entwicklungen in Deutschland. Wir erwähnen hier den Klassiker von Fritz Stern: The Politics of Cultural Despair: a study in the Rise of the German Ideology. London/Los Angeles: UCLA University Press 1961; Peter Gay: Weimar Culture. The Outsider as Insider. Harper-Collins 1968; Uwe Spörl: Gottlose Mystik in der deutschen Literatur um die Jahrhundertwende. Paderborn: Schöningh 1997; Christoph H. Werth: Sozialismus und Nation. Die Deutsche Ideologiediskussion zwischen 1918 und 1945. Opladen: Westdeutscher Verlag 1996. B. Zilly verweist zudem auf das neueste Buch von Georg Bollenbeck: Eine Geschichte der Kulturkritik. Von Rousseau bis Günther Anders. München: C.H. Beck Verlag 2007, ein facettenreiches Porträt, welches wie F. Stern den antidemokratischen Konservatismus präsentiert, der jedoch zu dieser Zeit nicht durchweg faschistisch war. 
on mit den ordinären neo-mythischen Symbolen der nationalsozialistischen Ideologie verwechselt wird, wodurch die falsche Assoziierung authentischer religiöser Gefühle mit der Sakralisierung von Macht und mit den Kulten von Kraft, Vitalität und der Aufopferung ermöglicht wird. ${ }^{11}$

Die defensive Geste und ihre raue Erklärung hätten die Aufmerksamkeit von Kritikern und Interviewern auf sich ziehen müssen. ${ }^{12}$ Sowohl die Übersetzungsvariante als auch die Begründung, die Meyer-Clason gibt, sind nicht hinnehmbar. Beides erfordert Ablehnung, die nicht nur auf die scharfe Kritik am «vermittelnden» übersetzerischen Modus beschränkt ist, die Chiqueto (berechtigterweise) äußert:

Die häufige Sorge des Übersetzers, dem deutschen Leser metaphysische Themen zu ersparen, was er in seinen Aussagen zu verstehen gibt, kann leicht in Frage gestellt werden, wenn wir von der Erkenntnis ausgehen, dass diese Nachsicht zugunsten des Publikums, genau wie in sprachlichwissenschaftlicher Hinsicht, vereinfachend ist. Der Übersetzer teilt nicht die ästhetischen Ideale der deutschen Schriftsteller seiner Zeit, zumindest nicht im Hinblick auf den Versuch, nach neuen Ausdrucksformen in der deutschen Sprache zu suchen. Für sie stellte die Suche nach neuen Formen den Versuch dar, die vom NS-Regime in sie eingelegten Laster auszuradieren. Für Meyer-Clason würde eine derartige Einsatzbereitschaft die Bedürfnisse der Übersetzung wohlwollend annehmen und ihnen entgegenkommen. ${ }^{13}$

Obwohl die Akademie nie auf die Begründungen von Meyer-Clason reagiert hat, gibt es gewisse Vorbehalte - sotto voce - zwischen Übersetzern und Kritikern. Erwähnenswert sind zum Beispiel die Beobachtungen von B. Zilly, die den Eindruck eines Freud'schen Versprechers in Meyer-Clasons oben erwähnter Rhetorik bestärken (das Ersetzen der rosianischen Metaphysik durch den regionalistischen Vitalismus, die dem nationalsozialistischen Neo-Darwinismus viel näher kommt).

11 B. Zilly assoziiert diesen Vitalismus mit H. Kayserling, der zum Kreis der konservativen Revolution gehört, deren herausragende Ideologen unter anderem A. Möller van den Bruck, Julius Langbehn und Ernst Jünger waren. «Ein weiterer Konservativer, der JGR beeinflusst haben könnte, ein Modephilosoph zwischen den Kriegen, ein Apostel der Intuition, der Wesensschau, des Lebensdrangs, der gana, einen Begriff, den er aus dem Spanischen übernahm, auch ein bisschen Blut und Boden ist da, glaube ich. Ein gewisser Antikartesianismus war im Gefolge Nietzsches Mode, auch Heidegger davon angesteckt. Aber natürlich hast Du recht, trotz aller konservativen Wurzeln und Tendenzen von JGR, der sich ja in der Tat nicht als Aufklärer verstand: als irgendwie verwandt mit der Nazi-Ideologie kann man ihn weder verstehen noch missverstehen, eher als Mystiker oder Esoteriker, ein bisschen wie auch Fernando Pessoa» (B. Zilly).

12 Vielleicht hat der Vorrang der engagierten Soziologie in der Literatur eine gewisse Intoleranz für Literaturen geschaffen, die auf religiöse Gefühle abzielen. Der Fokus auf die Politik hat zweifellos einen weiten toten Winkel und eine große Unempfindlichkeit für Autoren und Denker geschaffen, die das Erbe der deutschen Vorromantiker und des deutschen Idealismus überarbeiten. 13 Chiqueto Barbosa: Leituras de Grande sertão: veredas, S. 66. 
In E-Mail- und Telefongesprächen teilt Zilly meine Befremdung mit den übermäßig drastischen Übersetzungsoptionen, Vulgarismen mit militärischen und kriegerischen oder manchmal für die rosianische Welt zu derben Konnotationen:

Mehr als seine $[\mathrm{M}-\mathrm{C}]$ Spionagetätigkeit interessiert mich im Augenblick seine übersetzerische Arbeit, natürlich der Briefwechsel mit dem Meister und auch kritische Studien wie die von Fabio Chiqueto Barbosa. Dieser deutet an, bzw. CMC selbst deutet wohl an, dass er sich einige Freiheiten beim Übersetzen herausgenommen habe, um irrtümliche Assoziationen mit der Nazisprache zu vermeiden, weshalb er den Stil entmetaphysiert habe. Da bin ich skeptisch, das müsste man genauer untersuchen, eher habe ich den Eindruck, Spuren von Landserdeutsch bei CMC zu finden, wie «Knarre» und «verrecken», «Köter», überhaupt vulgäre und aggressive Wörter, krachlederne Kraftausdrücke der niederen Umgangssprache, die Riobaldo eigentlich ganz fremd sind. Nazideutsch ist das nicht, aber erst recht keine Distanzierung davon. Eher fühle ich mich an Trivialliteratur der 60er Jahre erinnert, Hans Helmut Kirst zum Beispiel. Aber natürlich fehlen auch Versatzstücke deutscher Bildungssprache nicht. Barbosa hat wohl recht, wenn er sagt, dass eben genau die sprachliche Erneuerung nach der Naziverluderung, welche sich die deutschen Schriftsteller nach 1945 auf die Fahnen geschrieben hatten, bei CMC fehlt: der Wunsch des Autors nach Distanz von Schablonen wurde mit weitestgehender, wenn auch z. T. schönklingender Schablonenhaftigkeit «bedient» (natürlich ein Bärendienst). ${ }^{14}$

Ohne diesen Punkt und die Missverständnisse, die im kurzen Akt von $\mathrm{M}-\mathrm{C}^{15}$ in dem von mir verbliebenen Raum auftauchen, näher erläutern zu können, wende ich mich nun dem zweiten Teil zu: den Entdeckungen der Historikerin Priscila Perazzo über den Übersetzer Meyer-Clason.

14 Im Originaltext hat die Autorin an dieser Stelle das deutschsprachige Originalzitat von B. Zilly eingefügt, was hier im Fließtext direkt übernommen wurde (Anm. d. Üb.).

15 An dieser Stelle bietet es sich an, einige Bemerkungen zum Neomystizimus, dem Nationalismus und dem Nationalsozialismus (den Nationalsozialismen) in Deutschland in den 1920er und 1930er Jahren hinzuzufügen. Siehe hierzu Wolfgang Beutin et. al. (Hg.): Deutsche Literaturgeschichte. Von den Anfängen bis zur Gegenwart. 3. überarbeitete Auflage. Stuttgart 1989, S. $385 \mathrm{ff}$. In diesem Band finden sich interessante Passagen über die ideologischen Unruhen in Deutschland während der ersten Hälfte des 20. Jahrhunderts. Solche Erklärungen sind umso wichtiger, als die brasilianische Öffentlichkeit, einschließlich der Akademie, die Entstehung der früheren ideologischen und ästhetischen Strömungen des Faschismus weitgehend ignoriert, deren Elemente der Nationalsozialismus sehr selektiv aufnimmt und vor allem die mystisch-ästhetischen Feinheiten des poetischen Denkens in schändlicher Weise aufgreift. M-C sollte sich an den immensen Unterschied zwischen dem rassistischen wie sozialdarwinistischen Vitalismus-Mythos des Nationalsozialismus und den Ideen von Denkern und Künstlern erinnern, die sich zu Dimensionen hin orientieren, die jenseits der Grenzen von Sprache und sozialem Diskurs liegen. Vgl. auch den Anhang. 


\section{Der historische Bericht von Priscila Perazzo}

Im März 1997 verteidigte Priscila Perazzo ihre Dissertation O Perigo Alemão e a repressão policial no Estado Novo (dt. Deutsche Gefahr und Polizeirepression in Estado Novo $)^{16}$ im Sozialgeschichtsprogramm der Fakultät für Philosophie, Philologien und Humanwissenschaften der Universität São Paulo (FFLCH-USP) unter der Betreuung von Prof. Dr. Maria Luiza Tucci Carneiro. Die Dissertation enthielt ein Kapitel über das Bild, welches die Polizisten des DOPS sich von einem typisch deutschen Spion gemacht hatten, der für das Spionagennetzwerk von Kollaborateuren und Sympathisanten des NS-Regimes tätig war. Dieses Netzwerk bot einen kontinuierlichen Strom verschlüsselter Informationen für das Dritte Reich in Brasilien (wirtschaftliche und ideologische Möglichkeiten sowie politische, kommerzielle und militärische Bewegungen in Häfen, mögliche zukünftige Zugänge von Reichsfreundlichen in Brasilien usw.). Seitdem ist bekannt, dass Meyer-Clason zu diesem Netzwerk gehörte und zu 20 Jahren Gefängnis verurteilt wurde (von denen er 5 in Ilha Grande absaß und dank einer Begnadigung durch das Militärgericht freigelassen wurde $^{17}$ ).

Am Prüfungskomitee nahm Dieter Strauss teil, der von 1996 bis 1998 Direktor des Goethe-Instituts in São Paulo war. Strauss zeigte sich überrascht und ungläubig, Meyer-Clason - den berühmten und verehrten Direktor des Goethe-Instituts in Lissabon - der Verlegenheit einer solchen Anklage ausgesetzt zu sehen. Priscila Perazzo, die bis dahin nichts von Meyer-Clasons literarischer Karriere nach seiner Begnadigung 1948 gewusst hatte, zeigte sich ebenfalls von der Enthüllung über den Angeklagten überrascht. Nichtsdestotrotz versuchte sie, sich mit Meyer-Clason in Verbindung zu setzen und seine Geschichte von ihm persönlich zu hören. Ihre Briefe blieben jedoch unbeantwortet. Meyer-Clasons Schweigen und die Verlegenheit über die Auswirkungen in den Medien veranlassten sie, die Nachforschungen aufzugeben. Als ich 2017 Kontakt mit ihr aufnahm, suchte sie den gleichen Widerruf des Urteils, welcher seit Ende der 1980er Jahre zum versöhnlichen Grundton in universitären Kreisen geworden war: Der Verdacht hing unerbittlich in der Luft, gleichzeitig verbreiteten sich die entgegenkommenden, gleichzeitig jedoch vage bleibenden Interpretationen zugunsten Meyer-Calsons. Was bis heute vorherrscht, sind entschuldigende Vermutungen über die Karriere von Meyer-Clason als jungem Geschäftsmann und Gefangenem des brasilia-

16 Priscila Perazzo: O Perigo Alemão e a repressão policial no Estado Novo, São Paulo: Divisão Arquivo do Estado 1999.

17 Nach der damaligen Gesetzgebung galt dieser Straferlass nur für Strafen unter fünf Jahren. Trotzdem wurde Meyer-Clason in die Liste der freigelassenen Häftlinge aufgenommen. 
nischen Geheimdienstes. Ich betone die apologetische Höflichkeit, weil die verfügbaren Daten, so dünn sie auch gesäht sind, von einer Natur sind, die ungeheure Neugier weckt - beinahe eine fiktionale Spannung, wie sie in einem guten Thriller vorkommt, oder in Guimarães Rosas Roman selbst, der ebenfalls voller Geheimnisse und Rätsel ist.

Denn Perazzos Buch 0 Perigo Alemão e a repressão policial no Estado Novo ${ }^{18}$ enthält neben dem Dossier mit den personenbezogenen Daten der Untersuchten auch fotografisches Material, das vom brasilianischen Geheimdienst gesammelt wurde. Das DOPS hielt dieses Material für ausreichend, um das typische Spionageprofil im Dienste der NS-Regierung zu entwerfen. Unglücklicherweise, für uns Literaten, war Priscila Perazzo vor allem an der Konstruktion der Vorstellungswelt der Polizisten des Neuen Staates interessiert, daher widmete sie sich nicht den dargestellten Charakteren und verzichtete auf die Überprüfung der Fakten, die hinter dem Porträt und der vom DOPS geschmiedeten Narrative liegen. ${ }^{19}$

Nichtsdestotrotz regen die Elemente, die im DOPS-Dossier auftauchen und die in Perazzos Buch wiedergegebene Auswahl davon, zum Träumen an. Wir sehen dort die Fotos eines großen, schlanken jungen Mannes mit englischem Dandy-Flair, mit ansprechender und verführerischer Aura. Aus der Autobiographie wissen wir, dass er der Sohn eines Major in der deutschen Armee, ein Veteran des Ersten Weltkriegs, war, dass seine Mutter Engländerin war und dass die Familie nach der Niederlage im Ersten Weltkrieg, dem Börsenkrach und der Inflation in den späten 1920er Jahren verarmt war. Es ist daher überraschend, den jungen Curt zu sehen, der das Gymnasium verlassen hatte und als Lehrling in irgendeine Firma eingetreten war, am Steuer eines eleganten offenen Wagens zu sehen, in SA-Uniform (!) in die Kamera lächelnd.

Es ist eine merkwürdige Kombination aus der weltlichen Eleganz des Fahrers, der 1936 auf der einen Seite die Möglichkeit hatte, seinen Wagen ${ }^{20}$ nach Brasilien zu bringen, und der Uniform der braunen Hemden (meist rekrutierte, arbeitslose

18 Perazzo: O Perigo Alemão e a repressão policial no Estado Novo.

19 Ich stimme der folgenden Bemerkung von Zilly zu, die auf die merkwürdige Verflechtung von Realität und Fiktion verweist: «Es ist interessant, dass die DOPS-Agenten beinahe wie Autoren eines Romans fungierten und den Autor in einer Art und Weise antizipierten, in der er sich später mit seiner eigenen Fiktion, dem Bild eines Dandy, eines reichen Mannes, mit einem guten Leben, eines Frauenhelds, ein bisschen frech, kein Nazi-Fanatiker, der aber vielleicht seinen Charme zu Zwecken benutzte, die er als patriotisch verstand, beinahe ein männlicher Mata-Hari.»

20 Um sich ein Bild davon zu machen, was es hieß, in Deutschland in den 1930er Jahren ein Auto zu besitzen: Fritz Sterns Vater, Arzt und Universitätsprofessor mit erfolgreicher Arztpraxis, hatte keine Mittel, um ein Auto zu kaufen. Fritz Stern: Five Germanies I have known. New York: Farrar, Straus, Giroux 2006, S. 86. 
und verärgerte Kleinbürger). Die Bilder wurden von DOPS-Agenten gesammelt und kommentiert. In dieser Darstellung werden Fragen und Hypothesen aufgeworfen, die zu einem Thriller-Drehbuch führen könnten. Ich gebe zu, dass diese grauen, unsicheren, mehrdeutigen Bereiche des Lebens des Übersetzers MeyerClason meine Vorstellungskraft in ähnlicher Weise beeinflussen wie die rätselhaften Andeutungen, die Rosa in seinen Erzählungen strategisch verbreitet.

Ich möchte mit der gleichen Neugier an den historischen Bericht um MeyerClason herangehen, die von mir Besitz ergriff, als ich Grande sertão: veredas las und die mich dazu brachte, folgendes zu fragen: Wie konnte Deodorinas Kindheit gewesen sein, wenn sie sich als der Junge Reinaldo-Diadorim verkleidete (oder vom Vater verkleidet wurde)? Wer war Mutter Bigri? Warum hat Riobaldo das Haus von Selorico Mendes verlassen und sich der Bande von Zé Bebelo angeschlossen? Wie erklären sich der Sadismus von Hermogenes und Ricardão jenseits von Riobaldos Offenbarungen sadistischer Grausamkeit, die von ihm Besitz ergriff? In der gleichen Weise kann ich meine Neugier in Bezug auf Meyer-Clason nicht unterdrücken, nicht, um ihn anzuklagen, sondern um das zu verstehen, was rätselhaft ist, widersprüchlich und seltsam suggestiv in den Berichten über ihn. Was für ein Mensch war Meyer-Clason in den Jahren 1925-1936? Warum hat der Junge die Schule ohne Abitur abgeschlossen? Wie ist er als Jugendlicher mit seinem Bedürfnis sich zu behaupten umgegangen? Wenn die vom DOPS gesammelten Bilder, die angeblich aus einem von Meyer-Clason nach Brasilien mitgebrachten Album stammen, authentisch sind: Was hat den jungen Mann, der mit dem von der Uniform verliehenen Selbstbewusstsein posiert und die Fotos mit nach Brasilien bringt, dazu gebracht, sich der SA anzuschließen? Mit welcher Tapferkeit und Effizienz schaffte es ein Lehrling, der zur Zeit des Börsenkrachs 1929 neunzehn Jahre alt war, in nur sechs Jahren Karriere zu machen und sich zwischen Paris, London und Lateinamerika zu bewegen? ${ }^{21}$

Mit welchen Errungenschaften hat der junge Geschäftsmann die Hindernisse der Inflation überwunden, seine Karriere gefestigt und die Mittel erhalten, um ein Auto zu kaufen, abgesehen von seinem Import nach Brasilien? Wie können die Berichte darüber verifiziert und erklärt werden, dass er nach London gereist war und für ein amerikanisches Baumwollunternehmen gearbeitet hatte (einer der

21 Die Frage drängt sich vor allem aufgrund unserer Erkenntnisse über die Repression auf, die 1933 mit starker staatlicher Kontrolle begann (feindlich gegenüber Kontakten mit dem Fremden, außer wenn sie die Interessen des Staates, der Elite der Regierung oder der SS betrafen): Man denke nur an die Korrespondenz Adornos und Benjamins, die die strenge Kontrolle aller Bürger durch die Autoritäten der Nazis verdeutlichen, die jeglichen internationalen Kontakt misstrauisch beäugten, der ihren Interessen und ihrer Aufsicht aus dem Weg gehen würde. 
Artikel erwähnt, dass er 1936 in Paris gearbeitet hätte, bevor er nach Brasilien kam)? Weder der Literaturkritiker noch der Historiker sollten sich mit dem vagen Bericht zufrieden geben, der in fast jeder Zeitung und Zeitschrift, wie etwa dem Piauí-Magazin, erscheint:

\begin{abstract}
Meyer-Clason wurde am 19. September 1910 in Ludwigsburg ${ }^{22}$ im Südwesten Deutschlands geboren. Er war der Sohn eines Offiziers der Armee, lebte als Kaufmann und handelte mit Baumwollimporten. Sein Büro brachte ihn 1936 im Dienst einer amerikanischen Firma nach Brasilien und er zog nach Porto Alegre. Er war 31 Jahre alt, als er 1942 festgenommen wurde und an das Ministerium für politische und soziale Ordnung in Rio Grande do Sul verwiesen wurde mit der Anschuldigung, am deutschen Spionagenetz für das Dritte Reich beteiligt zu sein. Er bekannte sich zu dem Plan - unter Folter, wie er später aussagte. Er wurde vom nationalen Sicherheitsgericht zu 20 Jahren Haft verurteilt.
\end{abstract}

Zu dieser Zeit hatte Getúlio Vargas Hitler gerade den Krieg erklärt. In Brasilien lebende deutsche Staatsbürger wurden von Teilen der Bevölkerung mit dem Nationalsozialismus assoziiert. Viele wurden wegen des Verdachts der Spionage festgenommen. Einige sammelten tatsächlich strategische Informationen für Deutschland, aber sie waren keine professionellen Spione. «Es waren Einwanderer, die aus patriotistischen Gründen dachten, sie sollten bei den deutschen Kriegsanstrengungen mitwirken», sagt die Historikerin Priscila Ferreira Perazzo. $^{23}$

Meyer-Clason bestritt die Anschuldigungen und überzeugte seine Freunde und Bewunderer von der Falschheit von «Verleumdung» und Folter, die ihn dazu veranlasst hätten, seine Spionagearbeit zuzugeben. Seitdem gilt das Narrativ, die der Übersetzer in seiner Autobiografie (dem Buch Äquator) festigte, und die Berichte von Kollegen, Freunden und Journalisten, deren Blick auf den unbestreitbaren Vorzügen von Meyer-Clason ruht, während die überzeugendsten Daten beiseite gelassen wurden:

«Es war im Gefängnis von Ilha Grande», schreibt Felipe Tadeu anlässlich des 100-jährigen Geburtstags von M-C im Jahr 2010, als Curt Meyer-Clason seine Liebe zur Literatur entdeckte, als er 1942 von der Polizei von Getúlio Vargas verhaftet wurde. Gegen ihn wog die Anklage, als Spion für das faschistische Regime gearbeitet zu haben. «Das sind reine Verleumdungen. Jeder, der die Fakten wissen möchte, kann meinen autobiografischen Roman Äquator lesen, dort wird alles gesagt», erklärte er dem Reporter. «Ich wurde nach der Folter gezwungen, ein Dokument zu unterzeichnen, ohne es lesen zu dürfen, eine Erklärung der Polizei mit der

22 Ludwigsburg ist heute für die folgende Institution mit endlosem Namen bekannt: «Zentrale Stelle der Landesjustizverwaltungen zur Aufklärung nationalsozialistischer Verbrechen.» 23 Bernardo Esteves: O Jagunço de Munich. In: Revista Piauí 67 (2012). Verfügbar unter http:// piaui.folha.uol.com.br/materia/o-jagunco-de-munique/. Letzter Zugriff: 30.1.2018. 
Absicht, mich zu verurteilen», sagte der Deutsche. Der Übersetzer lebte von Dezember 1936 bis Mai 1954 in Brasilien. ${ }^{24}$

In den meisten dieser Berichte überwiegt der lobende Ton auf die Karriere des Übersetzers und Kulturmittlers nach 1945, mit Fokus auf seinen politischen Einstellungen und seinem Kampf gegen Diktaturen:

«Ich bin beeindruckt, mit welcher Aktivität Meyer-Clason die Verbreitung brasilianischer, lateinamerikanischer, portugiesischer und spanischer Schriftsteller zu einer Zeit leistete, als dies auch eine äußerst politische Aktivität war, ein Kampf gegen Diktaturen in diesen Ländern. Wer die Portugiesischen Tagebücher (1979) gelesen hat, in denen Meyer-Clason selbst vor 1974 über seine Arbeit als Direktor des Goethe-Instituts in Lissabon berichtet, gewinnt einen guten Eindruck davon, wie Kultur und Literatur gemacht wurden, die zu jener Zeit zwangsläufig auch politische Akte waren», sagt Michael Kegler, ein deutscher Übersetzer des angesehenen Angolaners José Eduardo Agualusa, des Portugiesen Gonçalo M. Tavares und des Brasilianers Fernando Molica. ${ }^{25}$

Berthold Zilly kommt auch zu einer versöhnlichen und freundschaftlichen Sicht auf Meyer-Clason (obwohl er das Buch von Priscila Perazzo gelesen hat) und interpretiert die Spionage für die Nazis als die Einfalt eines Mannes ohne politische Vision:

Wie es scheint, war es mehr dummer, naiver und falscher Patriotismus, der CMC dazu brachte, die Rolle des Nazis zu übernehmen; ein Fanatiker oder Verbrecher war er nie; vorher ein Konservativer, ein pseudo-patriotischer Dandy, politisch ziemich naiv. Es scheint, dass es einige Tatsachen in den Spionagevorwürfen gibt; Auf der anderen Seite gibt es in Kriegszeiten immer eine sehr weite Vorstellung von Spionage; was er anscheinend getan zu haben scheint, war, die Schiffsbewegungen der deutschen Botschaft in Rio zu melden; und dies war nicht wirklich ein geheimes, fast für jeden sichtbares Nebensächliches, was jedoch in diesen erhitzten Zeiten Kriegsverbrechen war. $^{26}$

Weiter wägt Zilly ab: «Es ist davon auszugehen, dass sich M-C aus eigenem Antrieb und spontan der NSDAP-Partei außerhalb Deutschlands angeschlossen hat; dies zeigt zweifellos eine enorme Blindheit, aber dann akzeptierte er die Inhaftierung als Abzahlung und beging kein anderes Verbrechen.» ${ }^{27}$ Als er den vorliegen-

24 Felipe Tadeu: Tradutor alemão Curt Meyer-Clason completa 100 anos em 2010. Verfügbar unter: http://www.dw.com/pt-br/tradutor-alem\%C3 \%A3o-curt-meyer-clason-completa-100-anosem-2010/a-5246916. Letzter Zugriff: 30.1.2018.

25 Tadeu: Tradutor alemão Curt Meyer-Clason completa 100 anos em 2010.

26 Persönliche Äußerung per Email, im September 2017.

27 E-Mail-Konversation im September 2017. Vielen Dank an den Freund Bertold für die Erlaubnis zur Veröffentlichung des Inhalts. 
den Aufsatz kommentierte, fragte Zilly erneut, ob die Spionagetätigkeiten relevant seien:

Wir müssten fragen, ob sie tatsächlich schädlich für Brasilien sind oder deutschen Kriegshandlungen Vorteile bringen. Vielleicht hat M-C vom Patriotismus profitiert oder gehandelt, ohne Sympathie für die Nazis zu teilen. In jedem Fall stimme ich zu, dass die angeblichen Handlungen ein Rätsel darstellen, zu dessen Aufklärung sich M-C geweigert hat.

Zilly sieht darin typisches Verhalten von Gebildeten, sie «halten sich für unschuldig und geben nur die Verfehlungen $\mathrm{zu}$, die man ihnen nachweisen kann. Einfache Leute brechen oft unter der Last der Anklage zusammen». ${ }^{28}$

Es wird sehr deutlich, dass sich Kollegen und Leser verpflichtet fühlen, die ethische Integrität von Meyer-Clason zu retten und ihm zu vergeben. Ich möchte zügig einen anderen Standpunkt aus derselben Perspektive klären, bevor ich mit meiner Argumentation fortfahre und mit den Fragen, die mir noch relevant erscheinen. Weder befinde ich mich in der Position noch möchte ich über MeyerClason urteilen, ihn verurteilen oder gar ihm vergeben. Ich gehe zurück zu meinem Ausgangspunkt: Ich möchte die Puzzleteile, über die wir verfügen, zusammenzusetzen und ihnen mehr Präzision geben. Um zum Beispiel auf die Erhebungen von Priscila Perazzo zurückzukommen, sollten wir zumindest einige Fragen zu dem Fotoarchiv stellen, das Priscila in ihr Buch aufgenommen hat. In diesem Dossier sind mehrere Fotos zu sehen, die Meyer-Clason in SA-Uniform zeigen, und der Autor des DOPS weist darauf hin, dass Meyer-Clason in Deutschland zu den NS-Angriffstruppen gehörte. Mit anderen Worten: Meyer-Clason muss sich zwischen den späten 1920er Jahren und 1936 der SA angeschlossen haben, bevor er nach Brasilien kam. Als Mitglied dieser Miliz (die viele als blutrünstige Bande bezeichnen), musste er die schreckliche Säuberung zumindest zur Kenntnis genommen haben, die Hitlers radikale Gegner 1934 auslöschte, ein von Hitler, Goebbels und Himmler inszeniertes Blutbad gegen die SA von Röhm und den Flügel der unnachgiebigen Sozialisten (unter dem Vorwand, Deutschland vor dem «Röhm-Putsch» zu retten, begann ein angeblicher Putsch, die Erfindung der Hit-

28 Es erhebt sich aber auch die Frage: selbst wenn er intensiv Spionage betrieben hat, hat er wirklich Menschen geschadet, und hat er Deutschland, also den Nazis, kriegswichtige Vorteile gebracht, und Brasilien dadurch geschadet? Und hat er vielleicht des Geldes wegen Spionage betrieben? Weniger aus Nazi-Begeisterung oder irregeleitetem Patriotismus? Rätselhaft ist dieses Verhalten in jedem Fall, und leider hat er nichts getan, um dieses und seine Hintergründe aufzuklären. Das unterscheidet offenbar gebildete von eher ungebildeten, einfachen Angeklagten: die Gebildeten halten sich für unschuldig, und geben nur die Verfehlungen zu, die man ihnen nachweisen kann. Einfache Leute brechen oft unter der Last der Anklage zusammen und geben alles zu, manchmal mehr als die wirklich verbrochen haben. 
ler-Propaganda, mit dem Massaker gegen den SA-Gipfel und gegen einen Teil des Armeegipfels begann der Aufstieg der SS und die Nazifizierung der Streitkräfte). Wir stellen daher zumindest die Möglichkeit fest, dass Meyer-Clason möglicherweise nicht aus Naivität dem Spionagenetzwerk beigetreten ist und dass die vom DOPS gesammelten Elemente das Profil des jungen Meyer-Clason vor seiner Wandlung im Gefängnis zeigen könnten: das Profil eines Militanten der Elite des Nationalsozialismus. ${ }^{29}$

All das geht natürlich nicht darüber hinaus, Hypothese und Spekulation über die Anhaltspunkte zu sein, die bisher aufgetaucht sind. Zum Schluss gehe ich zurück zum Buch von Perazzo und zu den untertitelten Fotos, die den «Unteroffizier der CLASON-Reserve» zeigen, «der der Sturmabteilung (SA) angehörte. Wir sehen ihn hier entsprechend in Uniform.» ${ }^{30}$

Elemente wie diese können Fälschungen, Erfindungen, Täuschungen und Mythen sein, die einen schweren Kollateralschaden an der Erinnerung an MeyerClason verursachen. Perazzos Absicht bestand darin, die Art und Weise zu untersuchen, wie Vargas politische Polizei ihre «Erzählungen` über die Verbrechen, die sie untersuchte, konstruierte. Perazzos Text geht davon aus, dass die von der Polizei organisierte, dokumentierte Vereinbarung in den Akten eine Konstruktion des DOPS selbst gewesen sein könnte, um das Verbrechen, das sie untersuchte und unterdrückte, $\mathrm{zu}$ 〈erzählen〉. Eine weitere Untersuchung hinsichtlich dessen hätte mehr Erklärungen liefern können, als wir bisher haben. Ich ende mit der erneuten Aufstellung des Aufgabenkatalogs, den wir erfüllen sollten, und zwar schon deshalb, weil die Erinnerung an Meyer-Clason von den Antworten abhängt. Obwohl es keinen Zweifel daran gibt, dass die Nazis ein Spionagenetzwerk aufrechterhalten haben und Meyer-Clason mit diesem zusammengearbeitet hat, ist das von Perazzo veröffentlichte Material vorerst nicht ganz zuverlässig und fordert von uns Antworten auf die folgenden Fragen und Zweifel: 1) Sind die Fotos tatsächlich von Meyer-Clason? 2) Wann diente Meyer-Clason in der regulären Reichswehr der Weimarer Republik? 3) Wann war er Mitglied der SA? 4) Ist er der NSDAP bereits in Deutschland beigetreten? 5) Machte Meyer-Clason seine unternehmerische Karriere als self-made-man oder wurde er durch Verbindungen zur

29 Kommentar von B. Zilly: «Das ist auch möglich. Aber die These der Naivität ist nicht absurd. Sehen Sie, was er später über Diktaturen und andere Regime in Lateinamerika und anderswo schrieb. Jedes Mal, wenn er über Politik schreibt, ist alles vage, mehr oder weniger korrekt, auch etwas inkorrekt. Er hatte kein analytisches Auge für soziale und politische Situationen, er wusste viel, im Großen und Ganzen hatte er recht, aber alles blieb ungenau, und in den Einzelheiten oftmals falsch. Die Grenzen zum Fabulieren sind fließend. Das Emotionale diktierte ihm oft die Feder.»

30 Perazzo: O Perigo Alemão e a repressão policial no Estado Novo, S. $184 \mathrm{f}$. 
SA oder der NSDAP begünstigt? 6) Oder war es sein Geschäft als Unternehmer, das von der Bedeutung des Baumwollsektors profitierte? 7) Was wissen wir über diese Karriere? Was war voreilig? Er arbeitete als Auszubildender in mehreren Unternehmen (welche, wann, wie?) und baute in wenigen Jahren (zwischen 18 und 26 Jahren) internationale Geschäftsbeziehungen auf, wirkte (trotz Hyperinflation, Arbeitslosigkeit und Elend in Deutschland) in Paris, Argentinien und Brasilien; stimmt die Geschichte in der Geheimdienstakte, dass er einen derartig privilegierten Lebensstandard hatte, dass er ein Auto importieren konnte, als er nach Brasilien kam?

Weder die Kollegen, die die Möglichkeit hatten, die Autobiografie von MeyerClason (Äquator) zu lesen, noch die Tochter Juliane konnten diese Zweifel klären. Zilly antwortete auf meine Fragen, dass ich das Buch erneut lesen müsse, und erinnerte mich lediglich daran, dass diese Autobiografie Ähnlichkeiten und Affinitäten mit Thomas Manns Roman Bekenntnisse des Hochstaplers Felix Krull hatte. Auf dem Berliner Kongress im November 2018 lieferte Douglas Valeriano Pompey eine Reihe von Informationen über die literarische Bildung, die MeyerClason im Gefängnis unter der Obhut eines Mitgefangenen erhalten hatte, der ebenfalls deutschen Ursprungs war $^{31}$ und dessen ideologische, der nationalistischen Bewegung nahestehenden Neigungen Meyer-Clason beeinflusst haben könnten.

Wir fahren also in einem Nebel der Unklarheit fort, der Meyer-Clason als erstaunlichen Verdächtigen zeigt oder als verlorenen Naiven, der in einer düsteren, historischen Konstellation versunken ist. Viele Kollegen verteidigen ihn sehr glaubwürdig als großen Kulturvermittler. Das Spiegel-Magazin, in dem Priscila Perazzo interviewt wurde, sagte, es gebe im Bundesarchiv in Berlin keinerlei Dokumente, aus denen hervorgehe, dass Meyer-Clason Mitglied der $\operatorname{NSDAP}^{32}$ war. Taís Lucas sagte mir jedoch in persönlichen Gesprächen, sie habe mehrere belastende Dokumente gefunden, was wiederum neue Verdächtigungen aufkommen lässt, die geprüft werden sollten. Zum Schluss komme ich nochmals auf meinen ursprünglichen Vorschlag zurück, diese Bestandsaufnahme als Grundlage für zukünftige oder laufende Forschungen zu erwägen.

31 Siehe Douglas’ Aufsatz in diesem Band.

32 vgl. online: http://magazin.spiegel.de/EpubDelivery/spiegel/pdf/7810189. Letzter Zugriff: 30.1.2018. 


\section{Anhang}

Priscila Perazzos Masterarbeit ließ aufgrund der Fokussierung der diskursiven Konstruktion der «Deutschen Gefahr» durch die brasilianische Polizei wenig Raum für die Untersuchung des Unterschieds zwischen Fakten und Fiktion bei den Aktivitäten, die in den Dokumenten und Fotos, welche in den Durchsuchungen und Gefängnissen des DOPS gesammelt wurden, entdeckt wurden. Bei ihrem Master war das Bild des DOPS von Bedeutung, nicht die Wahrhaftigkeit der Fakten und der Menschen, die das NS-Spionagennetz bildeten. Nachdem die Öffentlichkeit in Deutschland und Brasilien schockiert reagierten, gab Priscila Perazzo ihre Studien auf. In dem Interview, das ich mit ihr geführt habe, macht sie geltend, dass die Akten Übertreibungen enthalten könnten, um Paranoia und Feindseligkeit gegen die deutsche Bevölkerung zu schüren, so dass die Darstellungsweise das Image von Meyer-Clason als gefährlichen Spion geformt haben könnte, obwohl er in Wirklichkeit nur ein naiver Patriot war.

Dieser Eindruck wird jedoch durch die Lektüre der jüngsten These von Taís Campelo Lucas geschwächt. Die Forscherin liefert weitere Details über den äußerst strengen und fast professionellen Rekrutierungsmodus des Spionagenetzwerks und zu den umfassenden und effektiv integrierten Aktivitäten der Kriegsmaschine - sowohl innerhalb der Regierung als auch der NSDAP und ihres rassistischen ideologischen Apparats. Ihr Werk Nazismo d'além mar: conflitos e esquecimento ${ }^{33}$ (dt. Nazismus von jenseits des Meeres: Konflikte und Vergesslichkeit) betrachtet die Professionalität des NS-Spionagenetzwerks und bringt etwas mehr Klarheit über die Mitarbeit von Meyer-Clason in diesem Netzwerk. So erwähnt sie, dass Meyer-Clason bereits vor seiner Karriere als Spion in Porto Alegre als Unternehmer in Recife eine falsche (englische) Identität angenommen hatte. Leider klärt Lucas jedoch nicht, wer auf Meyer-Clason verwiesen hat, um in der Firma Robertson \& Son zu arbeiten und ihm seine englische Identität gibt:

Der von Immers und Arnold angeworbene Vertreter war Hans Curt Werner Meyer-Clason, der nach Porto Alegre berufen wurde. Meyer-Clason war Spezialist im Baumwollhandel, war Mitglied der Angriffs-Truppen in Deutschland, war Reserveoffizier der Wehrmacht und war der NSDAP in São Paulo beigetreten. Zu Beginn des Krieges wurde er für die US-Firma Edward T. Robertson \& Son in Recife eingesetzt, wo er sich als Engländer präsentierte, bis er entlarvt wurde. In dieser Zeit erklärte er, dass er «aus reinem Patriotismus und Eigeninitiative seine illegalen Aktivitäten begann und Informationen über die Seeschifffahrtsrouten der

33 Taís Lucas: Nazismo d'além mar: conflitos e esquecimento. Porto Alegre: UFRGS 2011. 
englischen Kriegsflotte sammelte, was er alles der Deutschen Botschaft mitteilte, wenn er durch Rio de Janeiro fuhr.» ${ }^{34}$

Lucas' Interpretation legt daher nahe, dass Meyer-Clasons eigene berufliche Karriere Teil eines nationalsozialitischen Netzwerks in Brasilien war. Im Anschluss an seine Rückkehr nach São Paulo sei Meyer-Clason über seine wahrscheinliche Entlassung aufgrund seiner Staatsangehörigkeit informiert worden, was nach seiner Rückkehr von einer Argentinienreise im März 1940 geschehen war. ${ }^{35}$ Wieder ist unklar, wer diese Informationen weitergegeben hat - das Unternehmen selbst? Oder die Abwehr? Ob das Motiv, sich als Engländer auszugeben Teil der geheimen Arbeit als Spion war oder nur ein persönlicher Schutz gegen die wachsende Feindseligkeit gegen Deutsche; ob der Job in diesem Unternehmen eine strategische Platzierung für die Interessen der Nazis (Materialversorgung usw.) war?

Das Verhör berichtet, dass Meyer-Clason von Arnold «den Auftrag erhielt, nach Süden zu kommen, um hier der Informant des deutschen Spionage-Dienstes zu sein, dessen Pläne in Lateinamerika von Immers koordiniert wurden». ${ }^{36} \mathrm{Er}$ übernahm diese Aufgabe in Porto Alegre und arbeitete ab August 1940 für die Möbelfirma Casa Alemã Ltda.

Gleichzeitig übernahm er die Aufgabe, Rohstoffe für die deutsche Botschaft in Rio de Janeiro einzukaufen. Um diese Funktionen besser wahrnehmen zu können, beauftragte er die Firma «A Controladora», die den Exporteuren größere Sicherheit bieten sollte und eine größere Kontrolle über die gelieferten Waren hatte. ${ }^{37}$

Kommuniziert wurde, schreibt Lucas, mit «verschlüsselten Briefen, geschrieben mit unsichtbarer Tinte aus einer Tablette aus Pyramidon, Mikrofotografien und Geheimcodes.» ${ }^{38}$ In einer dieser Operationen passierte Meyer-Clason ein Ausrutscher, der die geheimen Aktivitäten in Gefahr brachte, weshalb er vorübergehend seine Spionagerolle verlor sowie seine engen und häufigen Beziehungen zum deutschen Konsulat von Porto Alegre. Das Konsulat wie die Botschaft dienten als direkt von der deutschen Regierung kontrollierte Arme und folgten auch den An-

34 Lucas: Nazismo d'além mar, S. 182-183. Die Daten stammen aus dem DOPS-Bericht. Porto Alegre, 20. Mai 1942. DOPS, Aktennummer 36.691: Spionage-Netzwerk in Rio Grande do Sul oder Nazismus in Rio Grande do Sul, AESP.

35 Lucas: Nazismo d'além mar, S. 182-183.

36 Ebda., S. 183 zum Bericht. Porto Alegre, 20. Mai 1942. DOPS, Aktennummer 36.691: SpionageNetzwerk in Rio Grande do Sul oder Nazismus in Rio Grande do Sul, AESP.

37 Ebda.

38 Ebda., S. 192. 
weisungen der Partei. Daher wurden häufige Gäste zu interessanten Personen des brasilianischen Geheimdienstes:

\begin{abstract}
Meyer-Clason zum Beispiel zog Aufmerksamkeit auf sich, indem er ein Kapital bewegte, das höher war als das Gehalt, das er als Mitarbeiter eines lokalen Unternehmens verdiente. Anfang September 1940 besuchte er die Werkstatt des Brillentechnikers Reinhold Vogel in der Rua dos Andradas in Porto Alegre und bat ihn, ein Stück Papier unter seinem Mikroskop zu untersuchen, auf dem sich wirtschaftliche Daten befänden. Vogel bat Meyer-Clason, am Abend zurückzukehren, doch er erschien nie wieder. Vogel beschloss, das Papier zu untersuchen, das aus einem verkleinerten Foto bestand, welches auf einem maschinenbeschriebenen Umschlag klebte, auf dem er einen Fragebogen zum Kauf von Öl, die Versandskontrolle, eine Bitte um Informationen über die Rationierung und entsprechende Verfügungen entschlüsselte. Nachlässigkeiten wie diese stellten die wirklichen Aktivitäten der Agenten bloß. Wegen dieses Fehlers wurde Meyer-Clason von Immers' Spionagenetzwerk suspendiert. ${ }^{39}$
\end{abstract}

Kapitän Immers war der Vorgesetzte von Meyer-Clason im deutschen Spionagedienst in Brasilien. Meyer-Clason gestand, dass er ihm zwischen 1940 und 1941 folgende Informationen zur Verfügung stellte: (a) Informationen über die Produktion von Rohstoffen, die möglicherweise für die Vereinigten Staaten oder England bestimmt waren, b) Nachrichten über die Bewegung der englischen Dampfer, ihre Sendungen, ihre Route, Tonnage und den Bestimmungsort, c) Daten zu Fleischlieferungen im Hafen von Rio Grande.

Eduard Arnold wurde von Meyer-Clason über Wirtschafts- und Handelsfragen sowie über eine «schwarze Liste» informiert, die genaueste Daten über politische Orientierung, arische Abstammung und Sympathien für den «Dreimächtepakt» von Mitgliedern lokaler Unternehmen enthielt, wie aus den beigefügten Listen ersichtlich wird..$^{40}$

Agenten der Abwehr hatten strenge Auswahlkriterien bei der Suche nach NSSpionen in Brasilien. Die Mitglieder mussten die deutsche Staatsangehörigkeit haben und zielten darauf ab,

Agenten unter den Mitgliedern zu rekrutieren, die möglicherweise bereits durch einen Wehrdienst ein Pflichtgefühl bewiesen hatten, die das Terrain kannten und einige Kontakte im Land hatten [...vor allem] im gewerblichen oder industriellen Sektor in Brasilien. [Auf diese Weise versuchte die Abwehr], die Wahrscheinlichkeit von Fehlern oder Ineffizienz zu minimieren. ${ }^{41}$

39 Ebda.

40 Ebda., S. 183 zum Bericht. Porto Alegre, 20. Mai 1942. DOPS, Aktennummer 36.691: SpionageNetzwerk in Rio Grande do Sul oder Nazismus in Rio Grande do Sul, AESP.

41 Ebda., S. 184. Bezieht sich auf Hilton's Informationen vgl. Stanley E. Hilton: Suástica sobre o Brasil - A História da Espionagem Alemã no Brasil. Rio de Janeiro: Civilização Brasileira 1977, S. 54. 
All diese Daten machen es unwahrscheinlich, dass Meyer-Clason nur ein naiver Patriot war. Er scheint eine relativ wichtige Rolle im Dienste des NS-Spionagenetzes gespielt zu haben. Diese Hypothese stärkt auch die beträchtliche Strafe, die er erhalten hat: 20 Jahre Haft, eine der höchsten Haftstrafen, auch unter Gefangenen wegen Spionage. Wir wollen im Vergleich die Verurteilung anderer aktiver Sympathisanten erwähnen, die ebenfalls Mitglieder der NSDAP waren, und neben politischen Versammlungen auch Rituale wie jenes Nazi-Begräbnis im April 1941 in Novo Hamburgo organisierten, während dessen sie von der brasilianischen Polizei festgenommen wurden. Die Strafen für diese Art von Aktivität waren minimal: «Günter Schinke, Steppe, Gerstl, Wenker, Knecht, Schmitt, Meyer und Triebsees wurden zu zwei Monaten Gefängnis verurteilt und zahlten eine Geldstrafe von 5:000\$000 (Réis, Anm. d. Üb.). Der Rest wurde freigesprochen. ${ }^{42}$

42 Ebda., S. 196. Schinke war der Vater von Günther Franz Heinrich, bis 1937 stellvertretender Leiter des Nazi-Zentrums von Novo Hamburgo und ehemaliger Ortsvorsteher der deutschen Arbeitsfront, und von Otto Emil, der Anführer des Santa Catarina-Kreises der NSDAP in Brasilien war und in Deutschland lebte. 
